\title{
Quark Contributions to Nucleon Momentum and Spin from Domain Wall fermion calculations
}

\section{S. N. Syritsyn*}

Lawrence Berkeley National Laboratory, Berkeley, CA 94720, USA

E-mail: ssyritsynelbl.gov

\section{J. R. Green, J. W. Negele, A. V. Pochinsky}

Centerfor Theoretical Physics, Massachusetts Institute of Technology, Cambridge, MA 02139, USA

E-mail: jrgreen@mit.edu, negele@mit.edu, avp@mit.edu

\section{Engelhardt}

Department of Physics, New Mexico State University, Las Cruces, NM 88003-8001, USA

E-mail: engelaphysics.nmsu.edu

\section{Ph. Hägler}

Institut für Kernphysik, Johannes Gutenberg-Universität Mainz,

Johann-Joachim-Becher-Weg 45, D-55128 Mainz, Germany

E-mail: haegler@kph.uni-mainz.de

\section{B. Musch}

Thomas Jefferson National Accelerator Facility, Newport News, VA 23606, USA

E-mail: bmusch@jlab.org

\section{W. Schroers}

NuAS, Stubenrauchstr. 3, 12357 Berlin

E-mail: Wolfram.Schroersefield-theory.org

\begin{abstract}
We report contributions to the nucleon spin and momentum from light quarks calculated using dynamical domain wall fermions with pion masses down to $300 \mathrm{MeV}$ and fine lattice spacing $a=0.084 \mathrm{fm}$. Albeit without disconnected diagrams, we observe that spin and orbital angular momenta of both $u$ and $d$ quarks are opposite, almost canceling in the case of the $d$ quark, which agrees with previous calculations using a mixed quark action. We also present the full momentum dependence of $n=2$ generalized form factors showing little variation with the pion mass.
\end{abstract}

The XXIX International Symposium on Lattice Field Theory - Lattice 2011

July 10-16, 2011

Squaw Valley, Lake Tahoe, California

\footnotetext{
*Speaker.
} 


\section{Introduction}

Nucleon structure calculations on a lattice are important for both testing QCD as the fundamental theory of quarks and gluons and making predictions and complementing experimental efforts that aim to measure the full three-dimensional picture of the proton and nucleon, and to explore the origin of the nucleon spin. Lattice calculations of nucleon structure are pursued by many groups, see $[1,2,3,4]$ for recent advances. In this work, we report results for quark momentum fraction, spin and orbital angular momentum from calculations with $N_{f}=2+1$ domain wall fermions. We also report previous $N_{f}=2+1$ calculations using a mixed (domain wall valence and Asqtad sea) quark action [5] with substantially increased statistics and reduced errorbars [10].

Table 1: Lattice ensembles used in this work. Uncertainties in the pion masses are dominated by the scale uncertainty.

\begin{tabular}{ccc|c|ccc|cc}
\hline \hline$L_{s}^{3} \times L_{t}$ & $m_{\pi}[\mathrm{MeV}]$ & $m_{\pi} L_{s}$ & Sep. $T / a$ & $a m_{\text {sea }}$ & $a m_{\text {val }}$ & $a m_{\pi}$ & \# confs & \# meas \\
\hline \multicolumn{7}{c}{ Mixed action $(a=0.1240(25)$} & $\left.\mathrm{fm}, a m_{\text {strange }}=0.050\right)$ \\
$20^{3} \times 64$ & $293(6)$ & 3.68 & 9 & 0.007 & 0.0081 & $0.1842(7)$ & 463 & 3704 \\
$20^{3} \times 64$ & $356(7)$ & 4.48 & 9 & 0.010 & 0.0138 & $0.2238(5)$ & 631 & 5048 \\
$28^{3} \times 64$ & $356(7)$ & 6.27 & 9 & 0.010 & 0.0138 & $0.2238(5)$ & 274 & 2192 \\
$20^{3} \times 64$ & $495(10)$ & 6.23 & 9 & 0.020 & 0.0313 & $0.3113(4)$ & 486 & 3888 \\
$20^{3} \times 64$ & $597(12)$ & 7.50 & 9 & 0.030 & 0.0478 & $0.3752(5)$ & 563 & 4504 \\
\hline \multicolumn{8}{c}{ Unitary domain wall $(a=0.0840(14)$} & $\left.\mathrm{fm}, a m_{\text {strange }}=0.030\right)$ \\
$32^{3} \times 64$ & $297(5)$ & 4.06 & 12 & 0.004 & 0.004 & $0.1268(3)$ & 616 & 4928 \\
$32^{3} \times 64$ & $355(6)$ & 4.86 & 12 & 0.006 & 0.006 & $0.1519(3)$ & 883 & 7064 \\
$32^{3} \times 64$ & $403(7)$ & 5.52 & 12 & 0.008 & 0.008 & $0.1724(3)$ & 528 & 4224 \\
\hline \hline
\end{tabular}

We perform calculations that span different values of lattice spacing, volume and pion masses, see Tab. 1. With the domain wall fermion action, we perform high-statistics calculations using $300 \mathrm{MeV} \lesssim m_{\pi} \lesssim 400 \mathrm{MeV}$, and with the mixed action using $300 \mathrm{MeV} \lesssim m_{\pi} \lesssim 600 \mathrm{MeV}$.

Quark momentum and angular momentum can be extracted from the matrix elements of the quark energy-momentum tensor between nucleon states:

$$
\begin{gathered}
\left\langle P^{\prime}\right| \bar{q}\left[\gamma^{\{\mu} i \stackrel{\leftrightarrow}{D^{v\}}}-\langle\text { trace }\rangle\right] q|P\rangle=\bar{U}\left(P^{\prime}\right)\left[A_{20}^{q}\left(Q^{2}\right) \gamma^{\{\mu} \bar{P}^{v\}}+B_{20}^{q}\left(Q^{2}\right) \frac{i}{2 M_{N}} \bar{P}^{\{\mu} \sigma^{\nu\} \alpha} q_{\alpha}\right. \\
\left.+C_{20}^{q}\left(Q^{2}\right) \frac{1}{M_{N}} q^{\{\mu} q^{v\}}-\langle\text { trace }\rangle\right] U(P),
\end{gathered}
$$

where $q=P^{\prime}-P, \bar{P}=\frac{1}{2}\left(P+P^{\prime}\right), Q^{2}=-q^{2}$ and $A_{20}, B_{20}$ and $C_{2}$ are momentum transfer-dependent $n=2$ generalised form factors (GFF). The forward value of $A_{20}(0)$ gives the quark momentum fraction inside the nucleon, while the combination of $A_{20}$ and $B_{20}$ gives the quark angular momentum [6]:

$$
\langle x\rangle=A_{20}(0), \quad J_{q}=\frac{1}{2}\left[A_{20}(0)+B_{20}(0)\right] .
$$

Note that the $B_{20}$ GFF requires calculations with non-zero momentum transfer. In this work we concentrate on $n=2$ GFFs, with $n=3$ GFFs reported elsewhere [8]. 


\section{Renormalization}

The energy-momentum operator in Eq. (1.1) is scale-dependent and its matrix elements have to be renormalized for comparison with experiment. For the domain wall ensembles, we calculate the renormalization constants using the Rome-Southampton method [7] following the RI'-MOM prescription. In this scheme, the in- and out-quarks of the pertinent vertex function have the same offshell 4-momentum $p=p^{\prime}$ which defines the scale of the renormalization point $\mu^{2}=p^{2}$. This scale must be in the window $\Lambda_{\mathrm{QCD}}^{2} \ll \mu^{2} \ll \Lambda_{\text {lat }}^{2}=a^{-2}$, i.e. in the perturbative region but small enough to avoid significant discretization errors. There are indications that such a window does not exist for the hybrid action ensembles [8], and we use the renormalization constants from lattice perturbative calculations [9]. Since the gauge field in hybrid action calculations was HYP-smeared [10], we believe that the 1-loop perturbative calculations are a reasonably good approximation, and in Sec. 3, we will see close agreement between calculations using perturbative and non-perturbative renormalization.

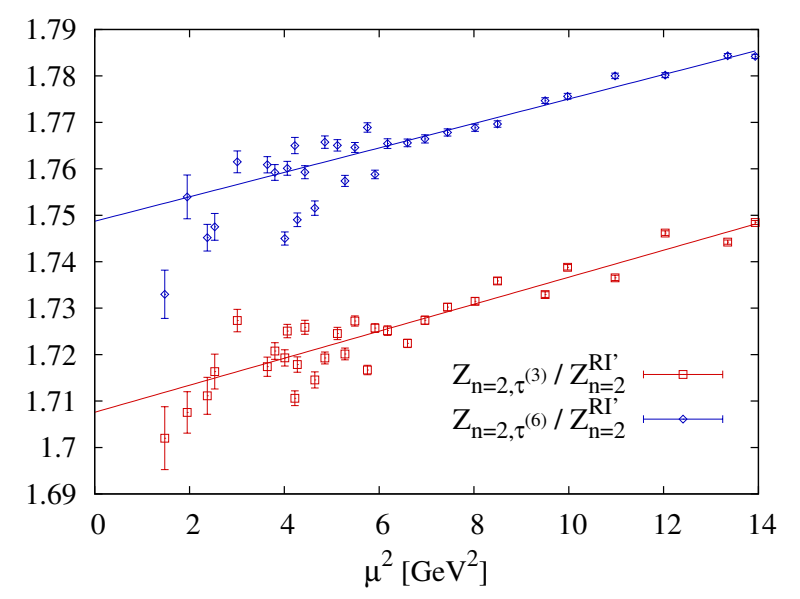

Figure 1: RI/MOM renormalization constant divided by 3-loop perturbative running (scale-independent). The extracted values must be multiplied with factors $Z_{A} / Z_{\mathscr{A}}$ and $\left(Z_{\mathscr{O}}^{\overline{\mathrm{MS}}} / Z_{\mathscr{O}}^{\mathrm{RI}}\right)_{2} \mathrm{GeV}$ to get the final renormalization constants.

We calculate vertex functions of the quark energy-momentum operator for two hypercubic representations $\tau^{(3)}$ and $\tau^{(6)}$ [11] and the (local) axial-vector current operator $A_{x, \mu}=\bar{q}_{x} \gamma_{\mu} \gamma_{5} q_{x}$. We use the latter to exclude the quark field renormalization, employing the fact that the conserved axial-vector current $\mathscr{A}_{x, \mu}$ of the domain wall fermions, with good precision, should have trivial renormalization $Z_{\mathscr{A}} \approx 1$, and calculating the ratio $Z_{\mathscr{A}} / Z_{A}$ from pion matrix elements separately.

In Fig. 1 we show the "scale-independent" renormalization constants, i.e., the lattice renormalization constants divided by the running constant $Z^{\mathrm{RI}^{\prime}}(\mu)$ calculated perturbatively up to 3 loops in continuum theory [12]. In the region $4 \lesssim \mu^{2} \lesssim 14 \mathrm{GeV}^{2}$, the "scale-independent" ratios vary within $\pm 1 \%$, demonstrating perfect matching between the lattice and the continuum renormalization factors. Assuming that the discretization errors contribute as $Z(a)=Z(0)+Z^{\prime} a^{2} \mu^{2}$, we extrapolate to $\mu=0$ and rescale our results to the final $\overline{\mathrm{MS}}(2 \mathrm{GeV})$ scheme. The final renormalization factors for the two representations are close to each other: $Z_{\tau^{(3)}}^{\overline{\mathrm{MS}}(2 \mathrm{GeV})}=1.708(4)$ and $Z_{\tau^{(6)}}^{\overline{\mathrm{MS}}(2 \mathrm{GeV})}=1.749(2)$. 


\section{Results}

To compute nucleon two- and three-point functions we use Gaussian-smeared sources tuned in Ref. [13]. The source-sink separation $T$ for both calculations is roughly $1.0 \ldots 1.1 \mathrm{fm}$ which we expect to be sufficient to suppress excited state effects at the precision level we aim at. We extract the matrix elements of the energy-momentum tensor using the standard ratio/plateau technique [14] and the overdetermined analysis $[13,14]$.
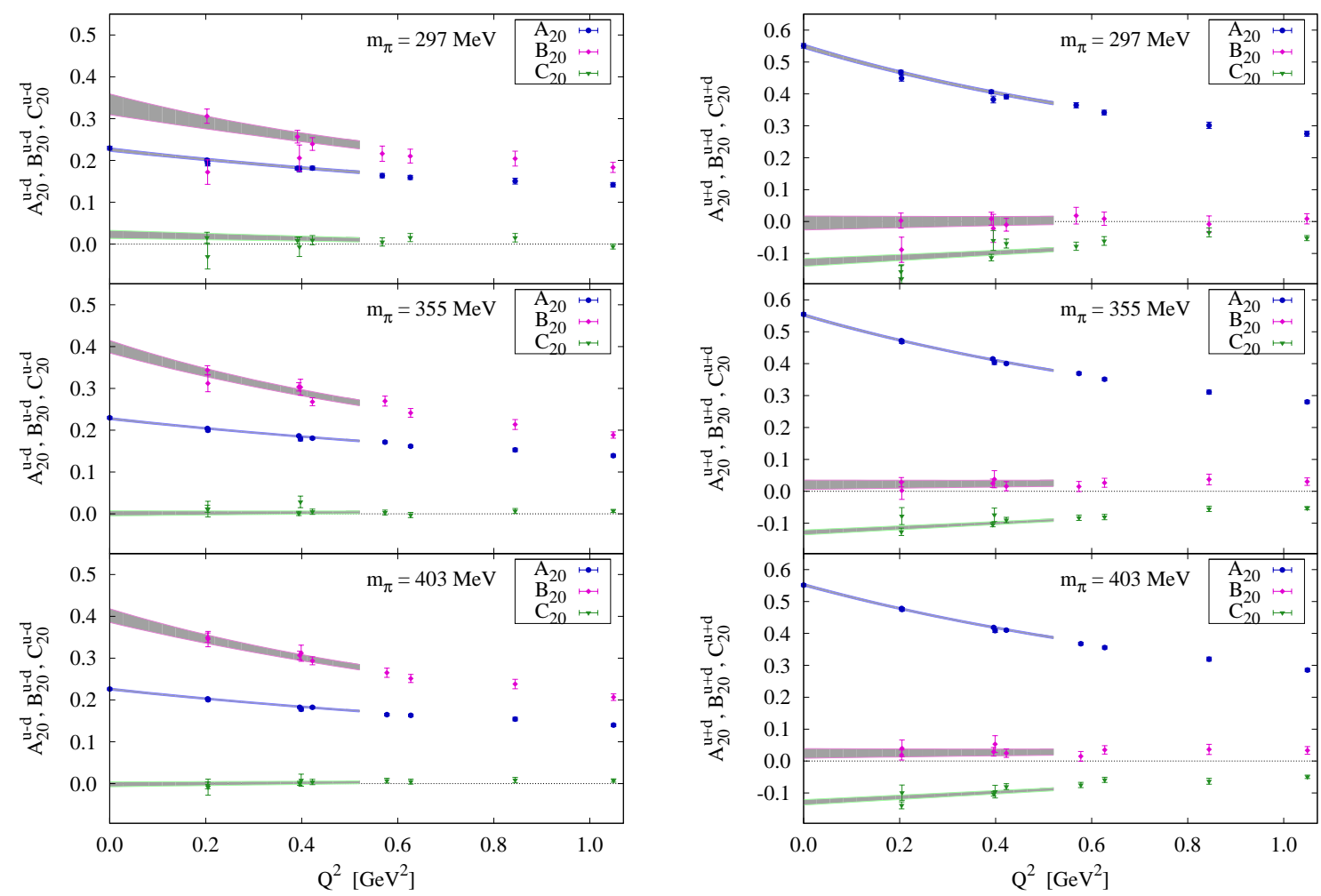

Figure 2: (Color online) Isovector (left) and isoscalar (right) $n=2$ Generalized form factors (GFF) of the proton from domain wall calculations.

In Fig. 2 we show the dependence of GFFs on the momentum transfer. The bands correspond to the dipole fits of each form factor and their statistical variation. Using these fits, we extract the forward $\left(Q^{2}=0\right)$ values of the isovector and isoscalar GFFs, as well as their slopes.

It is remarkable that there are specific features of the GFFs independent of $m_{\pi}$, e.g.

$$
\left|C_{2}^{u+d}\right| \gg\left|C_{2}^{u-d}\right| \approx 0, \quad\left|B_{20}^{u-d}\right| \gg\left|B_{20}^{u+d}\right| \approx 0
$$

which are also consistent with previous calculations [5] and large- $N_{c}$ counting rules [15].

We perform chiral extrapolation of the generalized form factors at $Q^{2}=0$ using baryon chiral perturbation theory [16]. While the formulas in Ref. [16] predict both the $m_{\pi}$ and $Q^{2}$ dependence, it is not clear if they are applicable for $Q^{2} \gtrsim 0.2 \mathrm{GeV}^{2}$. We therefore restrict our analysis only to the forward values and their derivatives obtained using the dipole extrapolation for $Q^{2} \rightarrow 0$. In Fig. 3 we show fitting and extrapolation results for the isovector GFFs. The analysis of the isoscalar GFFs 

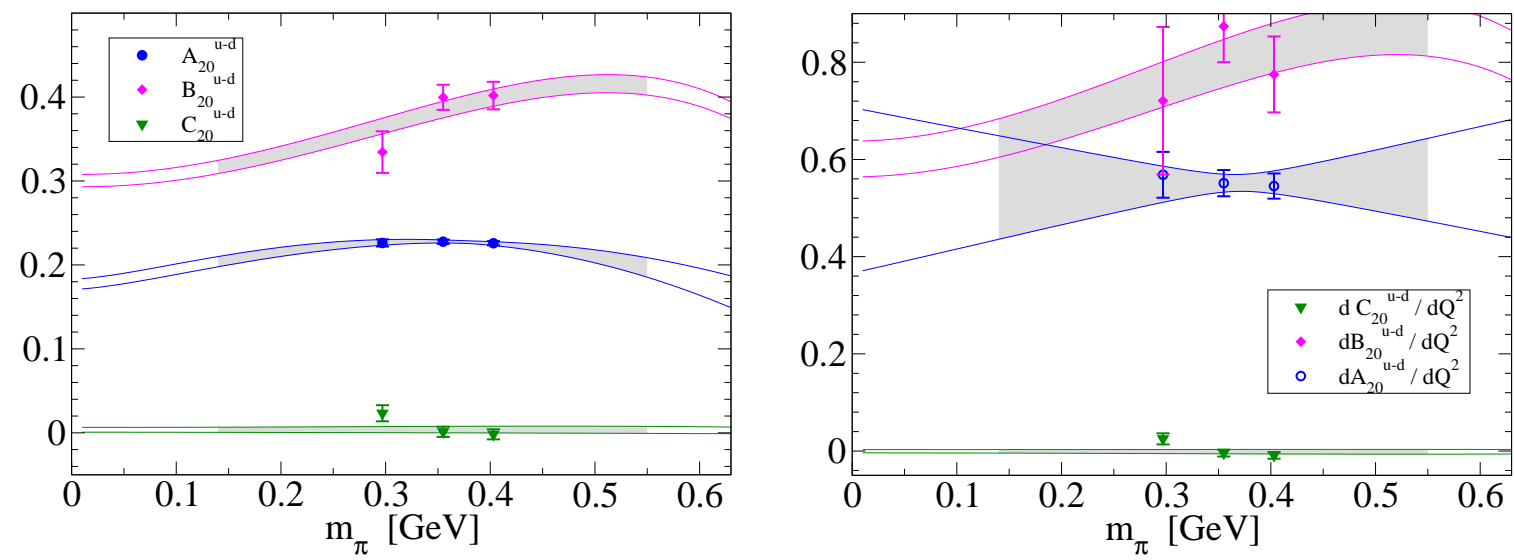

Figure 3: (Color online) Chiral extrapolations of isovector GFFs $A_{20}, B_{20}, C_{20}$ (left) and their derivatives $d / d Q^{2}$ (right) at $Q^{2}=0$. The ChPT predictions are taken from Ref. [16].
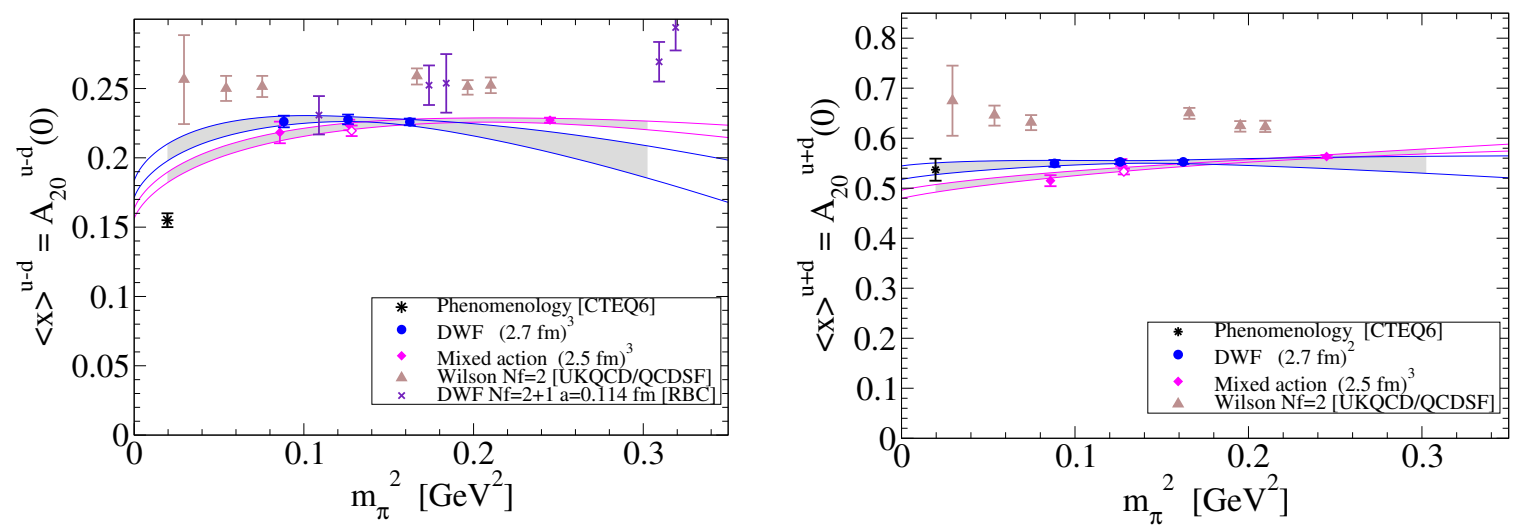

Figure 4: (Color online) Isovector (left) and isoscalar (right) quark momentum fraction in the proton compared to calculations in Ref. [1,2].

is similar. The GFF derivatives help to constrain the fits considerably, although less than the GFFs themselves.

In Fig. 4 we show our results for the quark momentum fraction $\langle x\rangle$ for both domain wall and hybrid action calculations. It is remarkable that the results agree very well, although lattice spacings and renormalization procedures are different. In addition we show the comparison of our results to those of Ref. [1] where $N_{f}=2$ Clover fermions were used and Ref. [2], which used $N_{f}=2+1$ domain wall fermions with $a=0.114 \mathrm{fm}$. It is interesting that $N_{f}=2+1$ domain wall results tend to agree at smaller pion masses, while $N_{f}=2$ Clover calculations disagree by $\approx 15 \%$. Our chirally extrapolated value of $\langle x\rangle^{u-d}=0.204(6)$ significantly overshoots the phenomenological value $0.155(5)$. This disagreement can be the result of either unreliability of the chiral extrapolation or presence of excited states in the matrix element calculation. At the same time, the isoscalar momentum fraction $\langle x\rangle^{u+d}$ agrees well with the phenomenology, albeit without disconnected contractions. This fact may be an indication of the smallness of the disconnected contributions.

In Fig. 5 we show quark angular momentum (disconnected contractions are not included), as well as separate contributions of $u$ and $d$ quark spin and orbital angular momentum (OAM) to the proton spin. Although not shown, results from the hybrid action calculations [10] agree very 
well with the data in Fig. 5(right). The calculations with domain wall fermions confirm previous qualitative results ${ }^{1}$ that

$$
\left|J^{d}\right| \ll\left|J^{u}\right|, \quad\left|J^{d}\right|=\left|S^{d}+L^{d}\right| \ll\left|S^{d}\right|,\left|L^{d}\right|, \quad\left|L^{u+d}\right| \ll\left|L^{u}\right|,\left|L^{d}\right| .
$$
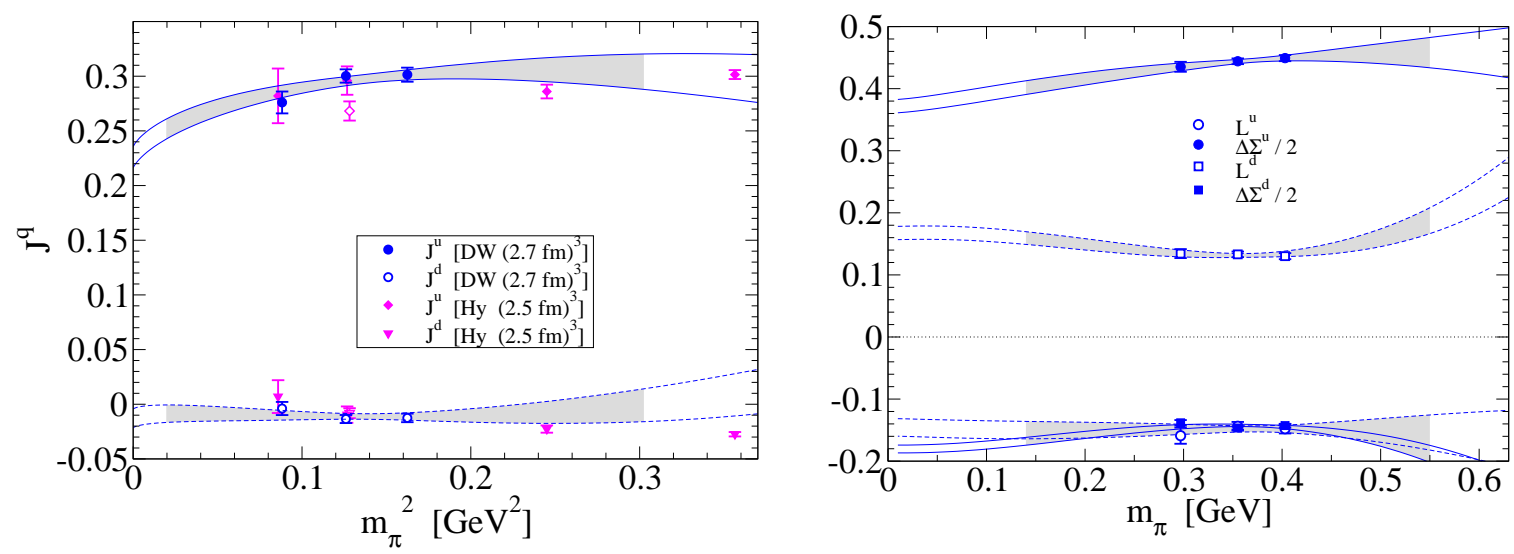

Figure 5: (Color online) Left: $u$ and $d$ quark contributions to the nucleon spin from the domain wall and hybrid action calculations. Right: $u$ and $d$ quark spin and orbital momentum from the domain wall calculations.

\section{Discussion}

We have computed the generalized form factors of the nucleon in fully dynamical QCD with $N_{f}=2+1$ flavors of chirally symmetric quarks. The pion masses $300-400 \mathrm{MeV}$ are still too heavy to be realistic, however, they are arguably close enough for the applicability of chiral perturbation theory. Nevertheless, we see disagreement between the extrapolated value of the isovector quark momentum fraction $\langle x\rangle^{u-d}$ and phenomenology. The presented agreement with the previous calculations employing different quark action, lattice spacing and operator renormalization procedure indicates that the problem may reside in either insufficiency of the order of the chiral perturbation theory expansion or presence of nucleon excited state contributions in the correlation functions. There are indications for excited state contributions in other calculations as well [17].

The disagreement with the $N_{f}=2$ calculations may also indicate that the number of flavors plays a crucial role for this quantity, leading to the conclusion that the low energy dynamics of QCD may affect $\langle x\rangle^{u-d}$ more than other quantities.

\section{Acknowledgements}

This research was supported in part by funds provided by the U.S. Department of Energy (DOE) under cooperative research agreement DE-FG02-94ER40818 and under Contract No. DEAC02-05CH11231. B.M. is supported by U.S. DOE contract No. DE-AC05-06OR23177. M.E. is supported by U.S. DOE grant No. DE-FG02-96ER40965. The U.S. Government retains a nonexclusive, paid-up, irrevocable, world-wide license to publish or reproduce this manuscript for U.S.

\footnotetext{
${ }^{1}$ We do not include the disconnected contractions, which can potentially modify Eq. 3.2.
} 
Government purposes. Computer resources were provided by the DOE through the USQCD project at Jefferson Lab, through its support of the MIT Blue Gene/L and through Argonne Leadership Computing Facility at ANL; and by the New Mexico Computing Applications Center (NMCAC) on Encanto.

\section{References}

[1] D. Pleiter et al, Nucleon form factors and structure functions from $N_{f}=2$ Clover fermions, POS (LATTICE2010) 153.

[2] Y. Aoki, T. Blum, H.-W. Lin, S. Ohta, S. Sasaki et al, Nucleon isovector structure functions in (2+1)-flavor QCD with domain wall fermions, Phys.Rev. D82:014501, 2010.

[3] T. Yamazaki et al, Nucleon form factors with 2+1 flavor dynamical domain-wall fermions, Phys. Rev. D79:114505, 2009.

[4] A. Lenz, M. Göckeler, T. Kaltenbrunner, and N. Warkentin, The Nucleon Distribution Amplitudes and their application to nucleon form factors and the $N \rightarrow \Delta$ transition at intermediate values of $Q^{2}$. Phys.Rev. D79:093007, 2009.

[5] Ph. Hägler et al, Nucleon Generalized Parton Distributions from Full Lattice QCD, Phys. Rev. D77:094502, 2008.

[6] Xiang-Dong Ji, Gauge invariant decomposition of nucleon spin, Phys. Rev. Lett. 78:610, 1997.

[7] G. Martinelli, C. Pittori, C. T. Sachrajda, M. Testa, and A. Vladikas, A General method for nonperturbative renormalization of lattice operators, Nucl. Phys. B445:81, 1995.

[8] LHP collaboration, Nucleon generalized form factors with domain wall fermions, in preparation.

[9] B. Bistrovic̀, Perturbative renormalization of proton observables in lattice QCD using Domain Wall fermions, $\mathrm{PhD}$ thesis, MIT, 2005.

[10] J. D. Bratt et al, Nucleon structure from mixed action calculations using 2+1 flavors of asqtad sea and domain wall valence fermions, Phys. Rev. D82:094502, 2010.

[11] M. Göckeler et al, Lattice Operators for Moments of the Structure Functions and their Transformation under the Hypercubic Group, Phys. Rev. D54:5705, 1996.

[12] J. A. Gracey, Three loop anomalous dimension of non-singlet quark currents in the RI' scheme, Nucl. Phys. B662:247, 2003; J. A. Gracey, Three loop anomalous dimensions of higher moments of the non-singlet twist-2 Wilson and transversity operators in the MSbar and RI' schemes, JHEP 10:040, 2006.

[13] S. N. Syritsyn et al, Nucleon Electromagnetic Form Factors from Lattice QCD using 2+1 Flavor Domain Wall Fermions on Fine Lattices and Chiral Perturbation Theory, Phys. Rev., D81(3):034507, 2010.

[14] Ph. Hägler et al, Moments of nucleon generalized parton distributions in lattice QCD, Phys. Rev., D68:034505, 2003.

[15] K. Goeke, M. V. Polyakov, and M. Vanderhaeghen, Hard Exclusive Reactions and the Structure of Hadrons, Prog. Part. Nucl. Phys. 47:401, 2001.

[16] M. Dorati, T. A. Gail, and T. R. Hemmert, Chiral Perturbation Theory and the first moments of the Generalized Parton Distriputions in a Nucleon. Nucl. Phys. A798:96, 2008.

[17] J. R. Green et al, Excited state contamination in nucleon structure calculations, POS (LATTICE2011) 157. 